\title{
Articulación de las tecnologías a través de la carrera Profesorado en Matemática de la Universidad Nacional de Rosario
}

\begin{abstract}
Resumen: En este artículo se analizan los modos de articulación de las tecnologías en las distintas actividades curriculares de una carrera de formación de profesores en Matemática de Argentina. Se consideran como marco de referencia los componentes del conocimiento disciplinar-pedagógico-tecnológico $\mathrm{y}$, mediante un enfoque cualitativo y diseño de estudio de caso, se aplica un cuestionario abierto online a los profesores de la carrera como primera fase de indagación. Entre las categorías de interés se encuentran las actividades con tecnologías, así como sus soportes y fundamentos dados por los docentes, aquellas experiencias especialmente valoradas con la relevancia que le otorgan para la formación de formadores $\mathrm{y}$, finalmente, algunas comparaciones emergentes a partir de la irrupción tecnológica debido a la situación de pandemia. En términos generales, ha sido posible constatar que las experiencias reportadas son muy variadas entre sí, algunas con un potencial a desarrollar y otras con prácticas sustanciosas que resultan distintivas.
\end{abstract}

Palabras clave: Tecnologías. Profesorado en Matemática. Formación Docente.

\section{Virginia Magali}

Bonservizi

Profesora de la Facultad de Ciencias

Exactas, Ingeniería y Agrimensura de

la Universidad Nacional de Rosario (UNR). Rosario, Argentina.

(iD) orcid.org/0000-0002-5251-0278

$\triangle$ virbonservizi@gmail.com

Natalia Fátima Sgreccia

Doctora en Humanidades y Artes (con mención en Ciencias de la

Educación). Profesora de la Facultad de Ciencias Exactas, Ingeniería y Agrimensura de la Universidad Nacional de Rosario (UNR). Rosario, Argentina.

(D) orcid.org/0000-0003-2988-7410

$\bowtie$ nataliasgreccia@gmail.com

Recibido en 30/01/2021 Aceptado en 15/02/2021 Publicado en 19/02/2021

\section{Articulation of technologies through the career Professor of Mathematics at the National University of Rosario}

\begin{abstract}
This paper analyzes the modes of articulation of technologies in the different curricular activities of a teacher training career in Mathematics in Argentina. The components of disciplinary-pedagogical-technological knowledge are considered as a frame of reference. An open online questionnaire is applied to the professors of the career as the first phase of inquiry, through a qualitative approach and case study design. Among the categories of interest, there are activities with technologies as well as their supports and foundations given by teachers, those experiences that are especially valued with the relevance they give for the training of trainers and, finally, some emerging comparisons from the technological irruption due to the pandemic situation. In general terms, it has been possible to verify that the experiences reported are very varied among themselves, some with a potential to be developed and others with substantial practices that are distinctive.
\end{abstract}

Keywords: Technologies. Teachers in Mathematics. Teacher Training. 


\section{Articulação de tecnologias por meio da carreira Professor de Matemática da Universidade Nacional de Rosário}

Resumo: Este artigo analisa os modos de articulação das tecnologias nas diferentes atividades curriculares de uma carreira de formação de professores em Matemática na Argentina. Os componentes do conhecimento disciplinar-pedagógico-tecnológico são considerados como um quadro de referência e, por meio de uma abordagem qualitativa e do desenho de estudo de caso, um questionário aberto online foi aplicado aos professores da carreira como primeira fase de investigação. Entre as categorias de interesse encontram-se as atividades com tecnologias bem como os seus apoios e fundamentos dados pelos professores, experiências essas especialmente valorizadas pela relevância que atribuem à formação de formadores e, por fim, algumas comparações emergentes da irrupção tecnológica devido à situação de pandemia. Em termos gerais, foi possível verificar que as experiências relatadas são muito variadas entre si, algumas com potencial para serem desenvolvidas e outras com práticas substanciais e distintas.

Palabras clave: Tecnologias. Professores de Matemática. Formação de Professor.

\section{Presentación}

Este artículo se encuadra en el Proyecto de Investigación La formación del profesor para desempeñarse en entornos de Educación a Distancia. El caso del Profesorado en Matemática (PM) de la Universidad Nacional de Rosario (UNR) (1ING584, 2018-2021, Argentina). En el marco de una Beca de Estímulo a las Vocaciones Científicas (Consejo Interuniversitario Nacional), hacia fines del 2020 se indagó al plantel docente de la carrera PM acerca de la conjunción de tecnologías de la información y la comunicación (TIC) en sus prácticas docentes en general y en ese año en particular.

Los objetivos específicos del Proyecto 1ING584 se resumen en reconocer necesidades formativas en el PM para la innovación educativa en materia de Educación a Distancia y Tecnología Educativa así como indagar acerca de la significatividad de los materiales producidos al respecto en ámbitos de formación docente.

Entre las investigaciones sobre el tema se destaca la preeminencia de estudios del uso del software GeoGebra en los distintos niveles educativos, con alcance de multiplataforma multi-referencial. Por ejemplo, Mántica y Freyre (2019) y Ramírez (2021) analizan experiencias que incluyen el uso de esta herramienta en el nivel superior, cuyos contenidos abordados se trabajan en la carrera de interés en esta investigación. En cuanto a los temas sobre los que se trabaja con GeoGebra, si bien pertenecen a diferentes áreas 
de la Matemática, Ward, Inzunza y Palazuelos (2021) reconocen que los profesores en Matemática prefieren temáticas relacionadas a la geometría para abordar con tecnologías y recursos digitales. En efecto, en función al estudio realizado, comentan que esto puede estar influenciado por la facilidad de acceso a este tipo de herramientas en dicha rama de la Matemática. Otra evidencia de la preeminencia de investigaciones que involucran al software GeoGebra es el trabajo reportado por Marques y Piveta (2020) donde se analizan las 14 producciones publicadas en la Conferencia Nacional de Modelización en Educación Matemática (CNMEM) desde 2003 a 2019 que mencionan a GeoGebra. En ellas, la modelización y el GeoGebra se conjugan ya sea con la formación de profesores o con aspectos propios del proceso matemático implicado o actividades puntuales en el marco de secuencias de enseñanza.

También ha ido en aumento el empleo de videos en las clases de Matemática (NEVES y BORBA, 2019), creciendo exponencialmente en la situación de pandemia del último año, en donde el proceso de elaboración comprende conjunciones epistemológicas, (inter)disciplinares, didáctico-pedagógicas, comunicativas, tecnológicas (SANTANA y JANUARIO, 2018) e incluso institucionales (AREA, 2009, 2020).

En una revisión de la literatura internacional en el campo de la Educación Matemática vinculado con la Tecnología Educativa, Borba et al. (2016) identifican cinco sub-áreas de investigación: tecnologías móviles, cursos en línea abiertos masivos, bibliotecas digitales y diseño de objetos de aprendizaje, aprendizaje colaborativo utilizando tecnología digital, capacitación docente mediante b-learning (BARTOLOMÉ, 2004). Al respecto reconocen avances en los últimos años, y delinean algunos desafíos y recomendaciones; entre estas: interpelar el aula de formación de los futuros profesores en Matemática en materia del uso especializado de las TIC, asunto que se asume en la presente investigación.

Por su parte, la carrera PM está radicada en la Facultad de Ciencias Exactas, Ingeniería y Agrimensura (FCEIA) de la UNR, en la ciudad de Rosario, al sur de la provincia de Santa Fe de la República Argentina. Existe desde hace más de tres décadas (ARGENTINA, 1988), con un último plan vigente desde el año 2018 (ARGENTINA, 2018), habiendo un cambio intermedio en el año 2002 (ARGENTINA, 2002).

Como se sostiene en el plan de estudios, su finalidad es "el desarrollo de recursos 
humanos con una sólida formación humanística, pedagógica, científica y tecnológica para el desempeño de la docencia en el área de la Matemática en los niveles de educación secundaria y superior universitaria y no universitaria” (ARGENTINA, 2018, p. 1).

Se procura contribuir a ello a través de cuatro años, u ocho semestres, mediante cuatro Campos de Formación:

- Disciplinar Específica (será abreviado en lo que sigue como CFDE, comprende 1968 horas, dentro de 19 actividades curriculares -la mayoría semestrales- en cuatro áreas: Análisis Matemático, Álgebra y Geometría, Matemática Aplicada, Educación Matemática).

- Pedagógica (CFP, 320 horas, tres actividades curriculares anuales).

- General (CFG, 240 horas, dos actividades curriculares anuales; además de un Examen de Suficiencia de Inglés).

- Práctica Profesional Docente (CFPPD, 544 horas, cuatro actividades curriculares anuales).

Puntualmente este trabajo tiene como premisa resignificar experiencias formativas de futuros profesores en Matemática cuando se emplean las TIC tanto en términos comunicacionales como didáctico-matemáticos. En específico, en esta ocasión, se relevan los modos de articulación de las tecnologías en las actividades curriculares de los distintos Campos de Formación del PM de la UNR.

\section{Encuadre conceptual}

Un modelo teórico para comprender prácticas docentes en Matemática es el del conocimiento matemático para la enseñanza (BALL, THAMES y PHELPS, 2008), o MKT (por su sigla en inglés: Mathematical Knowledge for Teaching), que los autores conciben como "un modo de construir puentes entre el mundo académico de conocimiento disciplinario y el mundo práctico de la enseñanza" (p. 398).

Precisamente, para desenmarañar la complejidad intrínseca de tales prácticas, se distinguen seis dominios de conocimiento, agrupados en dos grandes campos: conocimiento de la materia y conocimiento pedagógico del contenido. 
El conocimiento de la materia comprende, en primer término, un conocimiento común del contenido, que es utilizado en distintos ámbitos profesionales y científicos que se valen de la Matemática. Seguidamente, atendiendo a acciones específicas de la tarea de enseñanza, un dominio de conocimiento especializado del contenido, atravesado por la especificidad de la profesión del profesor en Matemática. También, un profesor requiere conciencia acerca de cómo los tópicos matemáticos están relacionados entre sí, con una visión holística abarcativa de las bases y fundamentos disciplinares.

Por su parte, el campo del conocimiento pedagógico del contenido amalgama el conocimiento del contenido con el conocimiento de los estudiantes (permite anticipar acciones, dificultades, errores y aciertos), el conocimiento de la enseñanza (incluye las formas didácticas de abordar el desarrollo de un contenido para hacerlo accesible a otros) y el conocimiento del currículum (vinculado con los programas diseñados para la enseñanza en cierto nivel educativo).

En esta instancia, al integrar el MKT con las TIC, se lo conjuga con el modelo teórico TPCK (por su sigla en inglés: Technological Pedagogical Content Knowledge) de conocimiento tecnológico pedagógico del contenido (MISHRA y KOEHLER, 2006), que aquí se denomina Conocimiento Tecnológico-Pedagógico-Matemático. Ambos sientan sus bases en Shulman (1986), quien advirtió sobre un tipo especial de conocimiento que los profesores poseen: pedagógico del contenido.

El TPCK supone que integrar las TIC en las clases implica no solo conocer las herramientas, sino también reacomodar las prácticas, esto es, revisar y resignificar los conocimientos pedagógicos y disciplinares cuando se incluye tecnología. Esa conjunción de conocimientos da origen, a su vez, a un nuevo conocimiento que es más que la suma de las partes (Tecnología + Pedagogía + Contenido).

En efecto, esta multirreferencialidad conlleva a que, además de considerar cada uno de esos componentes de manera aislada, se los considere en conjunción entre sí (Figura 1). Precisamente, estas interacciones entre dominios de conocimiento del profesor que trabaja con TIC se representan mediante la intersección de pedagogía y contenido curricular (conocimiento pedagógico disciplinar, o PCK), de tecnología y contenido curricular (conocimiento tecnológico disciplinar, TCK), de pedagogía y tecnología (conocimiento tecnológico pedagógico, TPK), y el que conjuga los tres elementos de 
conocimiento (T, P, C) simultáneamente: TPCK.

De este modo, al indagar acerca de qué conocimientos pone en juego un docente cuando enseña con tecnologías, en el modelo se consideran esos tres tipos articuladamente. Los profesores no solo necesitan desarrollar una flexibilidad cognitiva para cada uno de los tres conocimientos básicos componentes; sino que son convocados a comprender la manera en la que estos dominios interactúan mediante un equilibrio dinámico en pos a encontrar soluciones que sean sensibles a los contextos específicos.

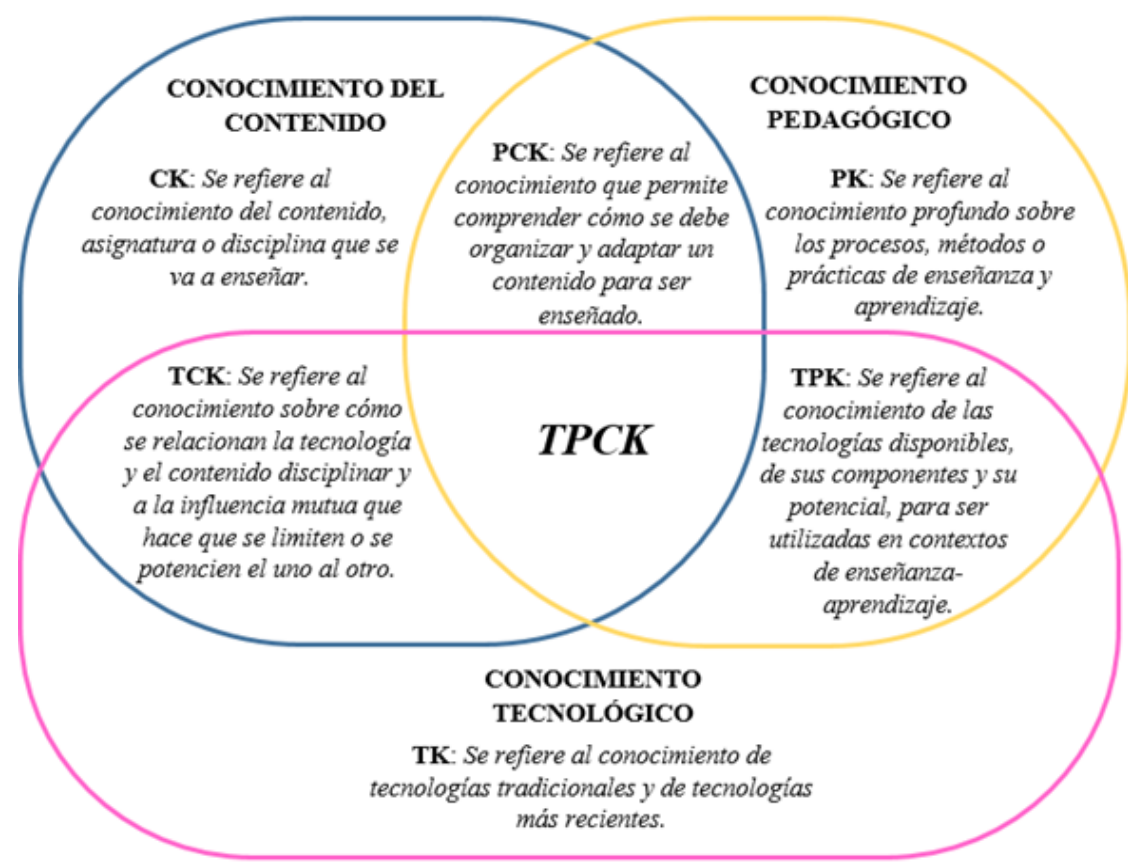

Figura 1: Modelo TPCK (BRUNINI, CHIRINO y DONATO, 2018, p. 122)

En el presente estudio se considera que asumir la generación de conocimiento acerca de las prácticas de la enseñanza de la Matemática cuando las mismas son pensadas y llevadas a cabo en entornos altamente tecnológicos no solo contribuye a fortalecer el conocimiento en cuestión del profesor (MKT, TCPK) sino que asume el compromiso social universitario (CECCHI, PÉREZ y SANLLORENTI, 2013) puesto en acción. Históricamente ciertas personas y poblaciones han visto limitadas sus posibilidades reales de concreción de su trayectoria de educación formal debido a formatos únicos y excluyentes. La modalidad de Educación a Distancia en las Universidades latinoamericanas, así como el enriquecimiento de vías de acceso a través de las TIC, emerge como una posibilidad de respuesta a ello (RAMA, 2006). 


\section{Enfoque metodológico}

El enfoque de la investigación es cualitativo debido a que interesa comprender las experiencias formativas de futuros profesores en Matemática en torno a las TIC en la carrera de interés: PM de la UNR. Procura estudiar la realidad en su contexto natural, centrando la indagación en los hechos, a fines de comprender los fenómenos de acuerdo con los significados que tienen para las personas implicadas (TAYLOR y BOGDAN, 1987).

Mediante el estudio se pretende captar la complejidad del caso en cuestión que, por sus características, puede dar lugar a resultados que, desde una lógica inductiva y sin ánimos de generalizar, proporcionen categorías válidas y útiles para analizar casos en situaciones semejantes (STAKE, 1995), como son carreras afines a Profesorados en Matemática, a nivel nacional y latinoamericano.

El diseño se plasma en tres fases a desarrollar durante un año. En este trabajo se comparte lo relativo a la primera de ellas, desarrollada en el primer semestre de ejecución, focalizada en relevar los modos de articulación de las tecnologías en las actividades curriculares de los distintos Campos de Formación del PM de la UNR.

Puntualmente se aplica un cuestionario abierto online (Formulario de Google) a cada integrante de los equipos docentes a cargo de las actividades curriculares, siendo 73 entre profesores y ayudantes.

En lo Cuadro 1 se muestran las categorías de interés del estudio asociadas a las cuestiones que se indagaron en esta fase de trabajo.

Cuadro 1: Categorías de análisis y preguntas específicas formuladas

\begin{tabular}{|l|l|}
\hline \multicolumn{1}{|c|}{ Categorías } & \multicolumn{1}{c|}{ Preguntas } \\
\hline $\begin{array}{l}\text { Actividades con TIC y y } \\
\text { Soportes tecnológicos }\end{array}$ & $\begin{array}{l}\text { ¿Qué actividades se realizan en la cátedra que impliquen la } \\
\text { utilización de TIC? ¿Qué soportes tecnológicos se utilizan? }\end{array}$ \\
\hline Fundamentos de selección & ¿Por qué fueron seleccionados dichos soportes? \\
\hline $\begin{array}{l}\text { Experiencias especialmente } \\
\text { valoradas }\end{array}$ & $\begin{array}{l}\text { Mencione alguna o algunas actividades que valore } \\
\text { especialmente. Sobre la/s misma/s comentar: ¿Cuáles fueron } \\
\text { los contenidos implicados? ¿En qué momento del desarrollo } \\
\text { del tema -introducción, desarrollo, aplicación, evaluación- se } \\
\text { abordó la actividad de referencia? ¿Cómo se dio el uso de TIC } \\
\text { por parte de los/as estudiantes? ¿Qué le implicó a usted como } \\
\text { docente antes, durante y luego del desarrollo de dicha }\end{array}$ \\
\hline
\end{tabular}




\begin{tabular}{|l|l|}
\hline & $\begin{array}{l}\text { actividad? ¿Por qué razón considera que se distingue del resto } \\
\text { de las actividades propuestas? Agregue los comentarios que } \\
\text { considere que ayudarán a comprender la experiencia. }\end{array}$ \\
\hline $\begin{array}{l}\text { Relevancia en la formación de } \\
\text { profesores en Matemática }\end{array}$ & $\begin{array}{l}\text { ¿En qué sentido sus prácticas con TIC en el PM adquieren } \\
\text { especial relevancia en la formación de profesionales que a su } \\
\text { vez enseñarán Matemática en un futuro? }\end{array}$ \\
\hline $\begin{array}{l}\text { Comparación con el uso de } \\
\text { TIC pre-pandemia }\end{array}$ & $\begin{array}{l}\text { Con respecto al uso de TIC, en términos comunicativos- } \\
\text { didácticos-matemáticos, ¿qué semejanzas y diferencias } \\
\text { encuentra con respecto a su desarrollo pre-pandemia? }\end{array}$ \\
\hline $\begin{array}{l}\text { Enriquecimiento para clases } \\
\text { presenciales }\end{array}$ & $\begin{array}{l}\text { ¿Qué considera oportuno trasladar a sus clases presenciales? } \\
\text { ¿Por qué? }\end{array}$ \\
\hline
\end{tabular}

Fuente: Elaboración Propia

Cada persona responde el protocolo de preguntas en el momento que lo considera oportuno dentro de un plazo dado de dos semanas, contando finalmente con la participación consentida y voluntaria de 47 personas, quienes representan la totalidad de las actividades curriculares de la carrera.

El procesamiento de la información se realiza mediante la técnica de análisis de contenido (ANDER-EGG, 2003), desarrollando sucesivas etapas de desmenuzamiento del material a la luz de las categorías de interés (Cuadro 1), con la selección oportuna de información recolectada como indicador que ilustra las ideas expresadas a través de las modalidades emergentes. En lo que sigue se recorren las categorías del estudio con una síntesis interpretativa de las respuestas de los docentes del PM de la UNR.

\section{Actividades con TIC y Soportes Tecnológicos}

Todas las asignaturas reportan el uso de recursos con TIC para sus clases. Particularmente solo dos de ellas no explicitan recursos relacionados a la comunicación virtual, aunque se supone por sus respuestas que sí los han utilizado. Esto último se considera que está fuertemente relacionado al contexto de pandemia, en el que las clases presenciales fueron suspendidas. Las respuestas de los docentes muestran que todos ellos han adaptado sus clases a la virtualidad permitiendo que los estudiantes continúen avanzando, de alguna forma, en la carrera en cuestión.

Dentro de los recursos mencionados que posibilitan la comunicación virtual, se destacan las videoconferencias y los campus virtuales. Las videoconferencias son 
nombradas por 24 de las 28 asignaturas del Profesorado, donde la gran mayoría le atribuye como funcionalidad el desarrollo de las clases. Algunos agregan la palabra sincrónicas dejando abierta la puerta a otro tipo de actividades que se pueden realizar para desarrollar clases de forma asincrónica. Por ejemplo, un docente de Análisis Matemático II enumera (cabe advertir que, acorde al enfoque metodológico adoptado, se comparten los testimonios tal como han sido expresados por los participantes, sin modificación gramatical; por otro lado, al final de cada testimonio se indica el Campo de Formación al que pertenece el espacio curricular así como el semestre o año - en caso de materias anuales — del plan de estudios en que se ubica):

Clases sincrónicas por videoconferencias, repositorio de materiales en aula virtual, consultas sincrónicas y asincrónicas, propuestas de actividades medidas por software (CFDE, $2^{\text {do }}$ semestre).

Además, una cantidad considerable de docentes mencionan que utilizan las videoconferencias para tener un momento de encuentro con los estudiantes. Como menciona Weber (2020), cuánto de sincrónico y de asincrónico en realidad va dependiendo de las intencionalidades y necesidades.

En Álgebra y Geometría II, iniciamos con un encuentro de presentación (sincrónico) en el cual cada estudiante se presentaba con cámara y audio (en la medida de lo posible), luego nos presentamos los docentes y explicamos las cuestiones generales relacionadas a la materia (CFDE, $2^{\text {do }}$ semestre).

Los campus virtuales son mencionados por 22 de las 28 asignaturas. Los docentes tienen a disposición dos opciones, una de ellas es a nivel Universidad (UNR, denominado Comunidades) albergando a otras 11 Facultades y tres Escuelas Secundarias, mientras que la otra es solo para la Facultad en cuestión (FCEIA: campus V). Ambas se apoyan en la plataforma Moodle y por lo tanto cuentan con herramientas similares. Desde la gestión institucional (tanto UNR como FCEIA) se procuró estar presente en este repentino proceso de virtualización (PAIN, GUINART y REYES, 2020) y se brindó acompañamiento a los docentes para el uso de estas plataformas y, también, la libertad de elegir la que deseen.

Con respecto a las funcionalidades de este recurso, los profesores destacan: compartir material; organizar trabajos prácticos, exámenes o tareas; interactuar en foros y realizar consultas, ya sean individuales o socializadas. A continuación se comparte un 
fragmento de la respuesta de un docente que explicita las funcionalidad del campus virtual en una materia de primer año.

En Análisis Matemático I es el espacio de Comunidades UNR (plataforma Moodle) el gran soporte tecnológico que alberga o referencia un link de acceso a los distintos apuntes, prácticas, ejercitación con herramientas de la plataforma, exámenes parciales y materiales audiovisuales con los que se desarrollan los contenidos (videos de exposición de contenidos y/o resolución de ejercicios, grabaciones de clases sincrónicas, links a material externo); y continúa siendo el principal medio de comunicación con los estudiantes, mediante los foros de Avisos o la mensajería del sistema (CFDE, $1^{\text {er }}$ semestre).

Al continuar con aquellos recursos que posibilitan la comunicación virtual, 13 de los sujetos encuestados mencionan el uso del email y de varios canales de chat como WhatsApp, Discord y Telegram, predominando WhatsApp por sobre los demás soportes que solo fueron mencionados por un participante en cada caso.

Por otro lado, se ha reportado el uso de recursos tecnológicos asociados al contenido o a lo pedagógico, más allá de lo comunicacional, en la mayoría de las asignaturas. De este modo, actualmente casi todas las materias del Profesorado usan TIC por razones que exceden a la necesidad de comunicarse impuesta por el contexto de pandemia.

Asignaturas de los cuatro Campos de Formación mencionan la utilización de presentaciones (Beamer, Power Point, Prezi) o videos con el fin de explicar contenidos o ejercicios. Por otro lado, en las actividades curriculares Recursos Tecnológicos en Educación Matemática, Práctica Profesional Docente III y Geometrías del Plano, son los propios estudiantes los que producen el material audiovisual, similar a la experiencia compartida por Santana y Januario (2018). Al respecto, un docente de Práctica Profesional Docente III comparte:

Todas las entregas y devoluciones de las actividades propuestas son mediante la plataforma institucional en formato de áudio y/o video a partir de foros. En entregas de actividades particulares se ha pedido a las estudiantes que generen algún material audiovisual como entrega final del Informe, sin un formato preestablecido (CFPPD, $3^{\text {er }}$

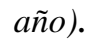

Los softwares matemáticos son consignados por 12 de las 19 asignaturas correspondientes al CFDE. Los mencionados son GeoGebra, Maple, Mathematica, Máxima, SAGE, Wolfram Alpha y uno específico para la asignatura "Modelos y 
Optimización" del cual se desconoce su nombre. El recurso GeoGebra fue el más reportado por una amplia mayoría de asignaturas, lo que guarda un correlato con lo plasmado en la presentación (apartado 1) acerca de la preminencia de estudios sobre el uso de dicho programa.

Otro recurso potente para los profesores en Matemática son los procesadores de texto LaTex, que son utilizados por los docentes del PM para la confección de apuntes. Lo que resulta interesante es que en las asignaturas Recursos Tecnológicos en Educación Matemática, Geometrías del Plano y Modelos y Optimización se proponen actividades en las que son los estudiantes (no solo los docentes) los que emplean esta base para la escritura matemática. En particular, las tres asignaturas mencionan como soporte Overleaf, dado que permite compartir plantillas y trabajar de forma colaborativa, como plantea un docente de la cátedra de Modelos y Optimización:

Otro soporte que se utiliza en esta materia es la plataforma de Overleaf (editor de Latex online y colaborativo) para organizar un trabajo en común de los estudiantes en la resolución de ejercicios (CFDE, $8^{\text {vo }}$ semestre).

Para finalizar con esta categoría de análisis, cabe advertir que Google Drive propició diversidad de usos. En asignaturas como Análisis Matemático I y Programación mencionan su uso solo como repositorio de material. En cambio, las actividades curriculares Práctica Profesional Docente I, Práctica Profesional Docente II, Geometrías del Espacio, Funciones Reales y Residencia aluden a la posibilidad que brinda de trabajar de forma colaborativa, en términos de comunidad virtual de práctica (GARCÍA, GRECA y MENESES, 2008), como se observa en este fragmento:

\begin{abstract}
Asimismo, el registro de experiencias de lo que acontece en el terreno donde los residentes llevan a cabo sus prácticas se realiza a través documentos compartidos en Google Drive lo que posibilita la continuidad en el acompañamiento/seguimiento entre clases presenciales (reemplazadas en el contexto de virtualidad por encuentros virtuales sincrónicos) (CFPPD, $4^{\text {to }}$ año).
\end{abstract}

\title{
5 Fundamentos de selección
}

Sobre los fundamentos de selección de los recursos digitales, 33 docentes ahondan al respecto. Puntualmente, 26 mencionan argumentos que priorizan la accesibilidad, contrarrestando una eventual brecha digital en pos a la inclusión digital (CABERO- 
ALMENARA y RUIZ-PALMERO, 2018). En palabras de un profesor de Álgebra y Geometría Analítica II:

Depende, algunos ya los conocíamos y otros fueron recomendados por pares. Las premisas fueron: universalidad (que la mayoría pueda acceder, por ejemplo, no utilizar una app que exista solo en iPhone, que es demasiado restrictivo), software libre, y/o que esté avalado y proporcionado institucionalmente (por ejemplo, las cuentas de Google Meet institucionales o el campus V de la FCEIA o la plataforma de Comunidades, etc.) (CFDE, $2^{\text {do }}$ semestre).

En este sentido, recalcan la gratuidad de los soportes, la practicidad de estos, el hecho de que sean promovidos por la institución y el uso previo de ellos, como puede advertirse en este testimonio (docente de Geometrías del Plano):

Para la selección de los softwares utilizados se priorizó el libre acceso y la familiaridad tanto de docentes como de estudiantes. [...] En particular, el software GeoGebra es el software más utilizado para construcciones geométricas, es libre, se puede usar online y es compatible con Moodle. Google Meet se utilizó porque la Facultad consiguió una cuenta permitiendo grabar las clases, almacenarlas y compartirlas a través de Google Drive, además las ventajas de Google Meet es que es más estable que Jitsi Meet y no tiene tiempo limitado como Zoom. Overleaf es una plataforma también libre donde se puede compartir y compilar archivos en LaTex (CFDE, $5^{\text {to }}$ semestre).

Como ejemplos de los soportes utilizados que son promovidos institucionalmente aparecen los campus virtuales tanto de la FCEIA como de la UNR, el correo electrónico del servidor institucional y Google Meet para las videoconferencias, dado que la Facultad puso a disposición cuentas de G Suite para el acceso a mayor cantidad de herramientas.

El campus virtual de la UNR resulta el soporte más utilizado previo a la suspensión de las clases presenciales. Además, se mencionan como ejemplos de recursos empleados con anterioridad a GeoGebra, WhatsApp, los correos electrónicos y los procesadores de texto LaTex.

Con la practicidad de los soportes se engloban respuestas que hablan de lo amigables que son determinados programas, de la facilidad para usarlos y de la popularidad de estos. En estos términos lo comenta un profesor de la actividad curricular Programación:

En general se priorizan aquellas herramientas de libre acceso y en lo posible de código abierto pero principalmente se seleccionan por su practicidad (CFG, $3^{\text {er }}$ semestre).

Por otro lado, 15 docentes expresan dentro de sus argumentos algún componente 
del TPCK (Figura 1). Los recursos son variados y de cada uno se mencionan características y posibilidades de uso. A modo de ejemplo, se muestra el siguiente fragmento:

La elección de Exam.net para la toma de exámenes finales escritos de Matemática Discreta en agosto se motivó en las siguientes característica positivas: su sencillez para armar una instancia de examen, la versatilidad del editor de texto incorporado donde cada estudiante escribe su resolución (que puede enriquecer con gráficos de GeoGebra u otra herramienta de dibujo tipo "Paint" también incorporada, en ambos casos, si la opción la habilita el docente), la posibilidad de subir fotos tomadas con un celular y su característica de control que apunta a que el estudiante no abandone la pestaña del examen en su navegador. Se puede complementar (y así lo hicimos) con el uso de cámaras web en una reunión en Google Meet si la cantidad de estudiantes no es excesiva. [...] otra característica que alentó el uso de esta herramienta es que el armado del examen es muy similar a lo que estamos acostumbrados: volcar una selección adecuada de ejercicios a un archivo pdf (CFDE, $5^{\text {to }}$ semestre).

\section{Experiencias especialmente valoradas}

Los profesores proporcionan experiencias muy variadas, que incluyen contenidos, momentos y tareas diferentes para todos los actores. Se advierte que, transversalmente, el trabajo colectivo docente (FELDMAN, 2020) ha resultado un sostén en este sentido.

Para evidenciar esta diversidad de experiencias, se comparten dos testimonios con actividades valoradas para momentos diferentes del desarrollo de un tema. El primero corresponde al de un profesor de la asignatura Análisis Matemático II, el cual aclara que experiencias como la que comparte se realizan para introducir cada nuevo tema.

Voy a tomar una como referencia: la introducción del concepto de integral impropia. La actividad consistía simplemente en considerar dos funciones, $f(x)=1 / x$ y $g(x)=1 / x^{3}$. En un primer momento se propuso esbozar la gráfica a mano. Se llegó a la conclusión que, sin entrar en detalles, ambas gráficas eran muy similares. Posteriormente se planteó que calcularan las integrales de $f$ y $g$ entre 1 y $x$, y dieran valores, muchos, cada vez más grandes, de la forma $10^{n}$. El objetivo era sacar conclusiones sobre qué ocurría con las integrales: en el primer caso, los valores de las integrales calculadas continúan creciendo a medida que x crece, en el segundo, en pocos pasos se estabilizan. Una vez. analizado este comportamiento, se pidió que realizaran las gráficas con el mismo programa, y las compararan. La gráfica exacta permite observar cómo la aproximación a 0 de $1 / x^{3}$ es mucho más "veloz" que la de $1 / x$, lo que permite que el área bajo la gráfica sea rápidamente "despreciable”. Una vez analizados estos ejemplos paradigmáticos, resulta sencillo y motivador introducir los conceptos de integral impropia convergente y divergente (CFDE, $2^{\text {do }}$ semestre).

El segundo relato que se comparte corresponde a un docente de Programación quien, dentro de las actividades desarrolladas en la materia, valora el momento de 
evaluación.

En particular representó un desafío la evaluación de la asignatura en el contexto de aislamiento producto de la pandemia. Esta instancia requirió una reflexión bastante profunda de los objetivos y las condiciones que son necesarias para cumplir dichos objetivos. Ha sido necesario plantearnos si tanto las TIC disponibles como nuestra propia formación nos brindaban los recursos necesarios para hacer un diagnóstico certero y justo del nivel de aprendizaje de les estudiantes. Finalmente, el resultado fue positivo pero requirió de un gran esfuerzo de coordinación e implementación de una metodología para todos (docentes y estudiantes) inédita (CFG, $3^{\text {er }}$ semestre).

$\mathrm{Al}$ explorar las razones por las cuales distinguen las actividades mencionadas, en 21 oportunidades se detallan argumentos relacionados con el contenido a abordar o con las decisiones pedagógicas que se tomaron para desarrollarlo. A modo de ejemplo, en la asignatura Geometrías del Plano se reporta la siguiente actividad:

Realizar unas construcciones con regla y compás, cada una en un archivo distinto de GeoGebra, ubicando en un costado los elementos datos, de forma que sea accesible modificarlos. El contenido implicado era construcciones con regla y compás, que contiene casi todos los conceptos desarrollados en la materia, rectas, segmentos, triángulos, ángulos, circunferencias, ángulos inscriptos en circunferencias, etc. La actividad también fue realizada en la etapa de aplicación de estos conceptos. Los estudiantes utilizaron el GeoGebra para dibujar los datos del problema (segmento y/o ángulo) y realizar paso a paso la construcción. Luego modificando estos datos pudieron observar cómo cambia la construcción e inferir condiciones para la existencia de solución para dicha construcción. Esta tarea es muy importante en esta materia porque engloba la esencia didáctica de la geometría y permite trabajar con todos los conceptos visualizándolos y analizando cómo se comportan (CFDE, $5^{\text {to }}$ semestre).

Por otro lado, 11 respuestas incluyen argumentos que se basan en el trabajo que realizaron los estudiantes o en lo que implicó o provocó la actividad en ellos. La respuesta que comparte uno de los docentes de Análisis Matemático II conforma un buen ejemplo este tipo de argumentos:

\begin{abstract}
Las propuestas que más valoro son aquellas en las que los estudiantes deben resolver una actividad de forma grupal para luego presentarla al resto de la clase. Se nota que los estudiantes asumen compromiso y responsabilidad con la actividad, hacen consultas y semana a semana se superan en el desarrollo y presentación de la misma (CFDE, $2^{\text {do }}$ semestre).
\end{abstract}

Algunos profesores, para dar las razones de la distinción de sus actividades, mencionan aspectos sobre su propia formación que se pusieron en juego al momento de planificar e implementar las actividades. En el siguiente testimonio de un profesor de Álgebra y Geometría Analítica II se puede observar cómo la valoración de la actividad se 
da por la posibilidad que brindó al equipo docente para reflexionar sobre sus propias interpretaciones.

Si tendría que elegir una actividad sería la propuesta en el Trabajo Práctico $N^{o} 1$ correspondiente a la unidad "Análisis combinatorio", me pareció interesante porque generó un gran debate entre los docentes de la cátedra, ya que hubo gran variedad de interpretaciones (CFDE, $2^{\text {do }}$ semestre).

Por último, con menor cantidad de apariciones, se encuentran aquellos aspectos mencionados sobre la relación del contenido a abordar y el aporte que puede hacer la actividad en un futuro docente. A modo de ejemplo, se menciona una actividad implementada en la asignatura Funciones Reales:

La actividad fue significativa pues se trabajaron aspectos de independencia en el estudio de nuevos conceptos matemáticos (apoyado en el trabajo previo desarrollado), el futuro rol docente (eligiendo, armando y justificando el uso de actividades) y la simulación del trabajo de un auxiliar de II en el equipo de cátedra valorando así el trabajo colaborativo en los equipo. En lo personal creo que fue una experiencia evaluativa enriquecedora, superadora del tradicional parcial de ejercicios para resolver, con la intención de fortalecer su formación como docentes de Matemática (CFDE, $7^{\text {mo }}$ semestre).

Como plantea Santaló (1999), amerita prestar atención a los modelos de enseñanza que los futuros profesores en Matemática viven en su formación, dado que sus prácticas y configuración del MKT estarán altamente permeadas por tales experiencias.

\section{Relevancia en la formación de profesores en Matemática}

Al analizar la relevancia que tienen las prácticas con TIC de los sujetos participantes del estudio en la formación de profesionales que a su vez enseñarán Matemática en un futuro, se han delimitado tres modalidades en las cuales se enmarcan las respuestas expuestas.

La más recurrente, Herramientas para la enseñanza, agrupa las que expresan que con sus prácticas con TIC están brindando herramientas y habilidades a los estudiantes para su enseñanza futura. En algunos casos destacan la posibilidad que tienen los profesores en formación de analizar el uso de TIC para la enseñanza, sus ventajas y desventajas. A modo de ejemplo, se muestra el siguiente testimonio (docente de Recursos Tecnológicos en Educación Matemática): 
Dado el contexto en el que vivimos, todo futuro profesor en Matemática debe estar preparado para enseñar en una sociedad cambiante. Las TIC forman parte de estos cambios, que repercuten en todos los ámbitos, y principalmente en la enseñanza. Desde el Taller no pretendemos que sean expertos en cada soporte que proponemos utilizar, porque como menciono, estos soportes cambian continuamente. Pero dada la heterogeneidad de experiencias previas en un primer año, pretendemos acercarlos a la tecnología y que comiencen a adquirir la capacidad de buscar herramientas, investigar su funcionamiento y proponer usos significativos en sus clases. Y digo que comiencen, porque creo que estas habilidades las irán desarrollando a lo largo de la carrera, y seguramente durante toda su vida profesional puedan mejorarlas (CFDE, $2^{d o}$ semestre).

De los 22 sujetos que aluden a esta idea, cinco expresan de alguna forma que las herramientas o habilidades se adquieren por la observación y otros dos aclaran que es a partir de vivenciar las experiencias como alumnos; ambas ideas se relacionan con la importancia de los modelos vividos durante la formación docente (SANTALÓ, 1999). Además, cabe destacar que algunos docentes resaltan que las tecnologías están en constante cambio y que las habilidades que los futuros profesores en Matemática desarrollen han de servirles para los recursos que estén disponibles en el futuro, como queda plasmado en el siguiente testimonio (profesor de Funciones Reales):

Me parece que es imprescindible que las TIC estén al servicio de la intencionalidad docente entonces si en la formación docente brindamos la posibilidad de experimentarlas y reflexionar sobre ellas desde una mirada crítica creo que estamos contribuyendo en la flexibilidad de sus futuras prácticas (en el contexto y con los recursos disponibles que existan) (CFDE, $7^{\text {mo }}$ semestre).

En segundo lugar, 18 docentes expresan argumentos que se apoyan en las Funcionalidades y ventajas del uso de TIC, las cuales se refieren tanto al ámbito educativo como más generales de desempeño de las personas. A modo de ejemplo, se puede observar el siguiente comentario de un docente de Residencia, en el que le otorga especial relevancia a un conocimiento situado del profesor — en términos de MKT — para optar por los recursos y materiales que considere óptimos:

\footnotetext{
Adquieren relevancia en tanto el uso crítico de las TIC es una de las habilidades de alfabetización básica que se requieren para la vida en sociedad hoy por hoy. En particular, es importante en la formación de profesores como profesionales que formarán futuros ciudadanos para sociedades cada vez más tecnologizadas; que tengan capacidad de explorar nuevas herramientas tecnológicas, evaluar su uso en cada contexto y situación particular, es también una decisión que le compete a un docente a la hora de seleccionar recursos y materiales para la enseñanza. Más específicamente en Matemática, pues hay softwares que potencian el desarrollo de habilidades matemáticas tales como la visualización, la exploración, la conjetura, entre otras (CFPPD, $4^{\text {to }}$ año).
}

Este fragmento de respuesta, además de hacer mención a la importancia del uso 
de TIC para desenvolverse en la sociedad y de explicitar algunas funcionalidades y ventajas de ciertos recursos para trabajar en las clases de Matemática, habla de la Importancia de la formación en el uso de TIC. Esta modalidad fue mencionada por otros 12 docentes más y en ella se agrupan respuestas que subrayan el rol protagónico de la carrera de Profesorado, de no indiferencia, ante la construcción del TPCK en los futuros profesores para integrar Matemática, Pedagogía y Tecnología.

\section{Comparación con el uso de TIC pre-pandemia}

Como semejanzas se menciona el uso de ciertos recursos tecnológicos, aunque en la mayoría de los casos expresan que no aprovechaban al máximo su potencial. Dentro de estos, el ejemplo más recurrente es el campus virtual, lo que coincide con lo reportado en la categoría Fundamentos de selección (apartado 5), en donde se lo referencia como el soporte más utilizado previamente. Además, se alude a los softwares matemáticos, Google Drive, el uso de un proyector para hacer presentaciones, el email, los procesadores de texto LaTex y los videos, con una pequeña cantidad de apariciones. Se puede observar parte de lo anterior en el siguiente testimonio, teniendo en cuenta que, en la asignatura en cuestión, el trabajo colaborativo está mediado por Google Drive (docente de Práctica Profesional Docente II):

Las semejanzas tienen que ver solamente con el trabajo colaborativo entre docentes y entre estudiantes y las entregas de trabajos por medio del Campus (CFPPD, $2^{\text {do }}$ año).

Aunque se exponen algunas semejanzas, las respuestas analizadas están enfocadas principalmente en las diferencias y más puntualmente en aquellos acontecimientos que se fueron desplegando a partir de la suspensión de las clases presenciales. El incremento en el uso de las TIC es lo más mencionado por los docentes. En efecto, 18 hablan de esto en sus respuestas; en algunos casos se refieren a recursos puntuales que eran utilizados con anterioridad, aunque con menor frecuencia o sin aprovechar todas sus herramientas. El ejemplo más mencionado es nuevamente el campus virtual. En la misma línea, 13 sujetos han reportado el uso de nuevos recursos y herramientas tecnológicas. Como ejemplos de estos recursos que comenzaron a utilizarse durante la pandemia, aparecen las videoconferencias y los videos, entre otros. Se puede observar una mención a la incorporación de nuevos recursos y herramientas en el siguiente testimonio (docente de 
Sujetos y Aprendizajes):

Entiendo que el contexto interpeló al ámbito educativo en torno al empleo de TIC y generó un debate importante. Además, se amplió la oferta de plataformas, que favoreció el avance de actividades en modalidad virtual (CFP, $3^{\text {er }}$ año).

Al seguir con aquello que sucedió a partir de la suspensión de las clases presenciales, aparece el propio aprendizaje que destacan los docentes en cuanto a los recursos tecnológicos. Esto fue tan mencionado como el uso de nuevos recursos. Para ilustrar esta idea, se muestra el siguiente relato de un docente de Análisis Matemático I:

En el desarrollo de las clases pre-pandemia considero que la utilización de las TIC era casi nula. Creo que la necesidad de la virtualidad en este nuevo contexto nos movió (y obligó) a explorar recursos y herramientas tecnológicas, por lo que resulta una experiencia de aprendizaje continuo en ese aspecto. Esta etapa es un constante indagar, implementar (prueba y error) y seleccionar las tecnologías más acordes y óptimas (CFDE, $1^{\text {er }}$ semestre).

Por otro lado, en algunas respuestas es posibles advertir cuestiones referidas a la oportunidad de usar diversas herramientas tecnológicas durante las clases, en cualquier momento/lugar, dado que para llevar a cabo una clase virtual los docentes cuentan con computadoras y la posibilidad de compartir sus pantallas con los alumnos. Se evidencia en el siguiente testimonio (profesor de Geometrías del Plano):

En este contexto el uso de las TIC fue más fácil y natural para aplicar ya que tanto los docentes como los estudiantes estaban conectados a un dispositivo con acceso a Internet donde podíamos utilizar GeoGebra o ver un video online en el momento que uno lo disponga durante la clase. Mientras que pre-pandemia esto era mucho más dificil de llevar a cabo (CFDE, $5^{\text {to }}$ semestre).

\section{Enriquecimiento para clases presenciales}

Las respuestas obtenidas acerca de esta categoría muestran que los docentes han descubierto en este año diversas herramientas que consideran interesantes para trasladar a las clases presenciales, aunque en varios casos se plantean dudas al respecto y una cierta incertidumbre por no saber cómo va a ser el desarrollo en un contexto diferente. Esto se puede observar en el siguiente parecer de un profesor de Pedagogía:

Habrá que ver con qué panorama nos encontramos el próximo año, pero seguramente 
muchas cosas van a quedar de esta experiencia. El uso del campus virtual va a ser una herramienta que, seguramente, seguiremos potenciando $\left(C F P, 2^{d o}\right.$ año).

La realización de consultas virtuales es una de las actividades que, según las respuestas de los docentes, llegó para quedarse aun cuando sea posible llevarlas a cabo de forma presencial. Específicamente fueron mencionadas en 13 oportunidades. Se distinguen dos formas de implementarlas, ambas con gran cantidad de menciones. La primera de ellas es mediante la interacción en foros, haciendo uso del campus virtual con el que cuente la actividad curricular. De este modo valoran que todos los estudiantes puedan ver las dudas y respuestas de los demás, evitando la duplicación de explicaciones. La segunda forma de implementar las consultas virtuales es mediante videoconferencias, de la cual destacan la posibilidad de asistir a las mismas sin tener que trasladarse y evitando las largas estadías en la Facultad debidas a la espera, puesto que usualmente las consultas presenciales son en contraturno. Además, algunos docentes están pensando en las videoconferencias para los trabajos grupales o para alguna otra actividad específica, más allá de los espacios de consulta. Se puede observar parte de lo anterior en la siguiente respuesta de un docente de Recursos Tecnológicos en Educación Matemática:

En base a la respuesta anterior, creo que en las clases presenciales mantendría el uso que le dimos a Comunidades, como así también consultas sincrónicas por Google Meet, más allá del encuentro semanal presencial. Estas consultas, años anteriores, eran presenciales en un horario a contraturno, lo cual a algunos estudiantes les demandaba un nиevo viaje a la Facultad o hacer tiempo después de otra materia. Asimismo, considero que la herramienta de videollamada será aprovechada por los estudiantes al momento de realizar los trabajos grupales (CFDE, $2^{\text {do }}$ semestre).

Como se evidencia en el testimonio anterior, los campus virtuales continúan apareciendo como grandes protagonistas, 10 docentes los han mencionado dentro de la categoría actual, en este caso expresan que aprovecharán mucho más sus herramientas con respecto al uso que hacían pre-pandemia.

Varios docentes del CFDE, precisamente ocho de un total de 30 que han hecho su aporte a esta categoría, expresan que utilizarían presentaciones en sus futuras clases presenciales; como ventajas mencionan que permiten agilizar y organizar mejor las clases. Un indicador al respecto se encuentra en las siguientes palabras de un profesor de Matemática Discreta: 
cañón en forma presencial. Disminuye el tiempo de escritura en pizarrón y permite explayar explicaciones (CFDE, $5^{\text {to }}$ semestre).

Otro de los recursos que planean trasladar a sus clases presenciales los docentes de dicho campo de formación son los softwares matemáticos. A modo de ejemplo, se muestra lo expresado por un profesor de la asignatura Análisis Matemático III:

Incorporaría presentaciones en Power Point o prácticas en GeoGebra para agilizar y sobre todo hacer más claros ciertos razonamientos que los alumnos deben aprender (CFDE, $3^{\text {er }}$ semestre).

Resulta oportuno destacar que algunos docentes expresan que continuarían con aquellas actividades que involucran a los estudiantes en el uso de TIC. Entre los ejemplos se mencionan la producción de presentaciones y videos, así como una actividad propuesta por la asignatura Modelos y Optimización en la que los estudiantes resuelven las prácticas de la materia de forma colaborativa utilizando un procesador de texto LaTex.

Finalmente, algunas respuestas muestran que se comienza a pensar en una modalidad mixta, en donde las instancias virtuales puedan complementar las clases presenciales. Se puede observar en el siguiente testimonio (docente de Álgebra y Geometría Analítica I y Modelos y Optimización):

Supongo que un sistema mixto va a permitir que algunos alumnos que no puedan asistir a clases por razones familiares, o económicas (que no viven en Rosario) van a poder estar haciendo carreras que no podrían en otro caso. Solo presencial para instancias más particulares o para evaluación (CFDE, $1^{\text {er }}$ y $8^{\text {vo }}$ semestres).

De este modo los docentes, en conjunto, han ido recuperando posibilidades de los entornos virtuales de aprendizaje que puedan, a su vez, fortalecer los entornos presenciales (LIBERMAN, 2020).

\section{Conclusiones}

En el trabajo se efectúa una aproximación a resignificar experiencias formativas de futuros profesores en Matemática cuando se emplean las TIC tanto en términos comunicacionales como didáctico-matemáticos, a través de la recuperación de los testimonios de los formadores de formadores. 
Puntualmente se contribuye con un relevamiento de los modos de articulación de las TIC en las actividades curriculares de los distintos Campos de Formación del PM de la UNR. Se ha podido constatar que las experiencias formativas de los futuros profesores en Matemática en torno a las TIC son muy variadas entre sí y con potenciales a seguir desarrollando. La variedad se puede observar en la cantidad de recursos y soportes reportados por los docentes, y fundamentalmente en las experiencias especialmente valoradas, sobre las cuales se ha intentado ilustrar esta diversidad a través de los fragmentos compartidos, quedando a su vez como un insumo valioso para continuar trabajando en la presente investigación. En el siguiente testimonio se observa que los propios docentes reconocen la posibilidad de continuar mejorando y potenciando las actividades (docente de Funciones Reales):

Este tiempo de Aislamiento Social, Preventivo y Obligatorio fue un tiempo de mucho aprendizaje, creo que hay aspectos en las propuestas que se deben mejorar, profundizar, reelaborar, pero que la utilización de TIC de manera crítica en la formación de profesores tendría que ser irreversible. Cuando nos referíamos a los cambios en Educación esos cambios los pensábamos lejanos casi meramente enunciativos creo que la pandemia nos mostró que tenemos que formar en el cambio y para el cambio, las TIC van a contribuir en ese sentido (CFDE, $7^{\text {mo }}$ semestre).

En sintonía con lo planteado por Gros (2016), los hallazgos indican la necesidad de reforzar las investigaciones que analicen y diseñen las prestaciones tecno-pedagógicas adecuadas para favorecer aprendizajes. Se ha visto que, en el PM de la UNR, a nivel institucional, se están promoviendo acciones a continuar reforzando en las prácticas habituales de la diversidad de Campos de Formación que esta carrera alberga. Se considera que una forma potente en este sentido es la socialización de las experiencias que se están llevando a cabo, lo cual se encuentra en la agenda de investigación del presente estudio.

Asimismo, esta primera fase de trabajo ha permitido identificar algunas prácticas docentes en la formación de profesores en Matemática que empleen las TIC de un modo distintivo. Entre ellas, las que involucran a los estudiantes en su uso, posibilitando que aprendan sobre las TIC y en muchos casos se coloquen en el rol docente al pensar cómo, a partir de las mismas, desarrollar clases o explicar ejercicios. Ejemplos de estas prácticas se mencionan en la categoría Enriquecimiento para clases presenciales (apartado 9) y se han reportado otras como la comentada por este profesor de Recursos Tecnológicos en 
Educación Matemática:

Es difícil elegir una actividad, porque creo que todas tienen lo suyo. Pero podría retomar la relativa a la definición formal de límite, la cual ha tenido más cambios de un año al otro. A partir de un video sobre el uso de GeoGebra, seleccionamos a modo de disparador una frase sobre la visualización de definiciones o propiedades abstractas, y elegimos la definición de límite como ejemplo de esto. En un foro, los estudiantes intercambiaron recursos que consideraban podrían utilizar para trabajar el tema como docentes. Este año, la mayoría propuso videos de YouTube y applets de GeoGebra. Realizamos una votación entre ellos, y nos quedamos con cuatro videos y cuatro applets para analizar. El análisis de los videos se llevó a cabo con PlayPosit, donde debían agregar comentarios y observaciones que les harían a sus alumnos si lo dejaran como material de estudio. En esto surgieron muchas dudas conceptuales con relación a la definición formal de límite. Para continuar "desarmando" y estudiando la definición, propusimos realizar de forma transversal a toda la actividad, un video colaborativo a nivel cátedra. La edición, a cargo de los docentes en un principio, y el material, a cargo de los estudiantes. Cada semana, dejábamos una definición coloquial e informal del límite de una función en un punto, la cual los estudiantes debían contradecir o dar un ejemplo de una función donde valía esto pero no la definición formal. Esta devolución debía ser en formato video, de 10 segundos. Se repitió algunas semanas, con contraejemplos cada vez más desafiantes, acercándonos a la definición que conocemos de límite y a la importancia de cada una de sus partes. Actualmente estamos con la edición final y lo utilizaremos, además, para incentivarlos a acercarse a algún programa de edición de videos y proponer mejoras sobre el mismo, antes de realizar su propio video de aplicaciones de la Matemática. Por otro lado, también analizamos, en primera instancia, qué ventajas y desventajas tenía cada applet. Luego, a partir de las distintas vistas y los protocolos de construcción, los estudiantes fueron construyendo su propio applet en sucesivas entregas. Finalmente, también con intercambios entre docentes y compañeros, plantearon y resolvieron actividades para trabajar con el applet de forma significativa. Estas actividades se realizaron colaborativamente en Overleaf. Como docentes, esta actividad nos implica siempre un gran desafío. Por un lado, las actividades y recursos propuestos van surgiendo de las entregas de los estudiantes, con lo cual la planificación es semana a semana. Continuamente surgen, por ejemplo, nuevos comandos o problemas con GeoGebra, que nos implica investigar y entender su funcionamiento, para decidir si es adecuado o no en el momento (y esto siempre junto al grupo, que realizan intercambios por el foro de consultas con sus propios compañeros y compañeras intentando hallar una solución o explicación). Por otro, con relación al video, con cada entrega debíamos pensar una forma de "retrucar" las respuestas para seguir incentivando en la búsqueda de contraejemplos por parte del alumnado (CFDE, $2^{\text {do }}$ semestre).

Con los docentes participantes de esas actividades curriculares se prevé realizar grupos enfocados en los que se profundizará sobre los aspectos de interés (fase 2). Posteriormente, en una tercera y última fase, se propiciarán innovaciones en la formación de profesores en Matemática que consoliden modalidades que se han ido introduciendo incipientemente a través de dispositivos con fuerte base tecnológica.

Trasversalmente, el hecho de estudiar cómo se configura un tipo particular de conocimiento (TPCK, MKT) en profesores en Matemática que se desempeñan en entornos con base tecnológica y de Educación a Distancia habilita distintas posibilidades 
de inclusión al sistema educativo. El campo social y pedagógico de la virtualización abre un abanico de vías de acceso a muchos docentes y estudiantes recreando formas alternativas de educabilidad, que promueven escenarios de enseñanza y aprendizajes compartidos en red.

Todo ello teniendo presente el compromiso social universitario en materia de docencia, investigación, servicio, extensión y transferencia de resultados, considerando la trascendencia de los profesores en la concreción del proceso de educación formal de las personas; en particular, de su alfabetización matemática. Qué hacen los docentes en sus clases marca indefectiblemente a generaciones. Ese hacer forma parte del conocimiento práctico del profesor. Aquí se lo conjuga con posibilidades de acceso y democratización de la mano de la Tecnología Educativa y, eventualmente, la Educación a Distancia.

Por otro lado, la Matemática es una de las áreas básicas rectoras de la Ciencia y la Tecnología. Una población matemáticamente alfabetizada estará en mejores condiciones de tomar decisiones con criterios racionales en tiempos de permanente cambio. A través de esta investigación se tiene la posibilidad de trazar acciones para promover una Educación Matemática en esta línea a fin de intercambiar saberes y experiencias.

Finalmente, se considera que los resultados de esta investigación constituyen una contribución para la Formación Docente en Matemática relativa a la modalidad, a fin de afrontar los retos y desafíos del tercer milenio, más allá de la situación de pandemia global. Esto requiere de profesionales formados en la disciplina Matemática y, al mismo tiempo, con experticia para trabajar con disponibilidad interdisciplinaria conjugando Didáctica y Tecnología Educativa. Más aún se necesita de quienes son, a su vez, sus profesores.

En términos de los dominios de conocimiento del TPCK y MKT, en conjunción entre sí (Figura 1), a partir de avances en el estudio compartidos, es posible reconocer una activación de todos ellos en clave de un compromiso social universitario que la emergencia demandó, procurándose mayores articulaciones en la medida en que las reflexiones y socializaciones se produzcan. Un trabajo colaborativo (HARGREAVES, 2020) para este tránsito se percibe como una de las claves. Como desafío se vislumbra que estas nuevas prácticas se acoplen y complementen las habituales en los entornos 
educativos universitarios (SCHLEICHER et al., 2020).

En específico, a partir de este estudio en el PM de la UNR se cuenta con una sistematización de un valioso material documental y experiencial al respecto, que puede ser útil también para otras carreras afines.

\section{Referencias}

ANDER-EGG, Ezequiel. Métodos y Técnicas de Investigación Social IV. Ciudad Autónoma de Buenos Aires: Lumen, 2003.

AREA, Manuel. Introducción a la Tecnología Educativa. San Cristóbal de La Laguna: Universidad de La Laguna, 2009.

AREA, Manuel. La transformación digital de la enseñanza universitaria en tiempos de covid-19, 2020. Video (67 min). Publicado por el canal Pedro Figueroa. Disponible en https://youtu.be/lElDoGUwjfE; acceso el 11 jun. 2020, a las 14h05.

ARGENTINA. Consejo Superior. Plan de Estudios del Profesorado de Enseñanza Media y Superior en Matemática. Rosario: Universidad Nacional de Rosario, 1988.

ARGENTINA. Consejo Superior. Plan de Estudios del Profesorado en Matemática. Rosario: Universidad Nacional de Rosario, 2002.

ARGENTINA. Consejo Superior. Plan de Estudios del Profesorado en Matemática. Rosario: Universidad Nacional de Rosario, 2018.

BALL, Deborah Loewenberg; THAMES, Mark Hoover; PHELPS, Geoffrey. Content knowledge for teaching: what makes it special? Journal of Teacher Education, Los Ángeles, v. 59, n. 5, p. 389-407, nov./dec. 2008.

BARTOLOMÉ, Antonio. Blended Learning. Conceptos básicos. Píxel-Bit, Sevilla, v. 23, p. 7-20, abr. 2004.

BORBA, Marcelo; ASKAR, Petek; ENGELBRECHT, Johann; GADANIDIS, George; LLINARES, Salvador; SÁNCHEZ, Mario. Blended Learning, E-Learning and Mobile Learning in Mathematics Education. ZDM, Hamburgo, v. 48, n. 5, p. 589-610, ago. 2016.

BRUNINI, Gladys; CHIRINO, Facundo; DONATO, Valeria. GeoGebra: un software paradigmático. En: SGRECCIA, Natalia Fatima. (Coord.). Procesos de acompañamiento en la formación inicial y continua de profesores en Matemática. Salamanca: FahrenHouse, 2018, p. 119-151.

CABERO-ALMENARA, Julio; RUIZ-PALMERO, Julio. Las tecnologías de la información y la comunicación para la inclusión: reformulando la brecha digital. International Journal of Educational Research and Innovation, Sevilla, n. 9, p. 16-30, ene. 2018. 
CECCHI, Néstor; PÉREZ, Dora; SANLLORENTI, Pedro. Compromiso Social Universitario. De la universidad posible a la universidad necesaria. Ciudad Autónoma de Buenos Aire: IEC-CONADU, 2013.

FELDMAN, Daniel. Algunas notas para una situación no esperada, 2020. Video (68 min). Publicado por el canal CITEP UBA. Disponible en https://youtu.be/1FYTQPGI3Zw; acceso el 8 abr. 2020, a las 12h30.

GARCÍA, Jesús; GRECA, Ileana; MENESES, Jesús. Comunidades virtuales de práctica para el desarrollo profesional docente en Enseñanza de las Ciencias. Revista Electrónica de Enseñanza de las Ciencias, Vigo, v. 7, n. 2, p. 439-462, may. 2008.

GROS, Begoña. Retos y tendencias sobre el futuro de la investigación acerca del aprendizaje con tecnologías digitales. RED, Murcia, n. 50, p. 1-13, jul. 2016.

HARGREAVES, Andy. Profesionalismo colaborativo. Cuando Enseñar Juntos supone el Aprendizaje de Todos, 2020. Video (82 min). Publicado por el canal Magisterio TV. Disponible en https://youtu.be/-EeY19CKS2A; acceso el 10 sep. 2020, a las 17h10.

LIBERMAN, Daniela. Enredarnos: interactuar para aprender en los EVEA, 2020. Video (66 min). Publicado por el canal CITEP UBA. Disponible en https://youtu.be/EHRyDpxlWcI; acceso el 8 jun. 2020, a las $20 \mathrm{~h} 23$.

MÁNTICA, Ana María; FREYRE, Magalí. Análisis de la relación entre imagen y definición en una situación problemática mediada por GeoGebra a partir de no ejemplos del concepto de poliedro regular. Educación Matemática, Ciudad de México, v. 31, n. 1, p. 204-234, abr. 2019.

MARQUES, Jorge; PIVETA, Wellington. Focos das pesquisas publicadas na CNMEM: Modelagem Matemática e GeoGebra. Educação Matemática Debate, Montes Claros, v. 4, n. 10, p. 1-21, 2020.

MISHRA, Punya; KOEHLER, Matthew. Technological pedagogical content knowledge: a framework for teacher knowledge. Teachers College Record, Nueva York, v. 108, n. 6 , p. 1017-1054, jun. 2006.

NEVES, Liliane; BORBA, Marcelo. Análise do discurso multimodal de um vídeo com conteúdo matemático. Educação Matemática Debate, Montes Claros, v. 3, n. 9, p. 220235, set./dez. 2019.

PAIN, Oscar; GUINART, Mildred; REYES, Maribell. Virtualización de emergencia/COVID-19, 2020. Video (63 min). Publicado por el canal Observatorio de Innovación Educativa. Disponible en https://youtu.be/aF2UNStAk5M; acceso el 04 jun. 2020, a las $19 \mathrm{~h} 30$.

RAMA, Claudio. La Tercera Reforma de la Educación Superior en América Latina y el Caribe. Ciudad Autónoma de Buenos Aires: Fondo de Cultura Económica, 2006.

RAMÍREZ, Bolívar. GeoGebra en 2D y 3D como recurso didáctico en un curso de integración múltiple: una experiencia de enseñanza-aprendizaje. Matemática, Educación 
e Internet, Cartago, v. 21, n. 1, p. 1-27, sep. 2020/fev. 2021.

SANTALÓ, Luis. La formación de profesores de Matemática para la enseñanza media. En: SANTALÓ, Luis; OTTOLENGHI, Carla; TRICARICO, Hugo; HERNAIZ, Ignacio; MARBACH, Perla; CHOUHY, Margarita; GARCÍA, Elena; MARMORATO, Mariel; GRECO, María Beatriz; GÓMEZ, Germán; GALAGOVSKY, Lydia; CETKOVICH, Teresita; FAURING, Patricia. Enfoques: hacia una didáctica humanista de la Matemática. Buenos Aires: Troquel, 1999, p. 209-214.

SANTANA, Rogério; JANUARIO, Gilberto. Ensino de Matemática e a produção de videoaulas. Educação Matemática Debate, Montes Claros, v. 2, n. 6, p. 254-264, set./dez. 2018.

SCHLEICHER, Andreas; CREAMER, Monserrat, ANGULO, María Victoria; ÁLVAREZ-TRONGÉ, Manuel; SPROSS, Verónica; HARGREAVES, Andy; BURD, Leo; CABROL, Marcelo; PÉREZ-ALFARO, Marcelo. una oportunidad para transformar: la educación latinoamericana y los desafíos post COVID-19, 2020. Video (115 min). Publicado por el canal Banco Interamericano de Desarrollo. Disponible en https://youtu.be/A7iSzkkRG-o; acceso el 23 jul. 2020, a las 20h05.

SHULMAN, Lee. Those who understand: knowledge growth in teaching. Educational Researcher, Los Ángeles, v. 15, n. 2, p. 4-14, feb. 1986.

STAKE, Robert. Investigación con estudio de casos. Madrid: Morata, 1995.

TAYLOR, Steve; BOGDAN, Robert. Introducción a los métodos cualitativos de investigación. Ciudad Autónoma de Buenos Aires: Paidós, 1987.

WARD, Silvia; INZUNZA, Santiago; PALAZUELOS, José. Uso de recursos digitales por profesores de matemáticas en secundaria: un estudio exploratorio. Matemática, Educación e Internet, Cartago, v. 21, n. 1, p. 125-153, sep. 2020/mar. 2021.

WEBER, Verónica. ¿Por qué elijo el encuentro sincrónico para mis clases?, 2020. Video (132 min). Publicado por el canal Secretaría General Académica. Disponible en https://youtu.be/TY20ps4Masw; acceso el 11 ago. 2020, a las 21h20. 